\title{
Sliding Modes in Constrained Systems Control
}

\author{
Asif S̆abanović, Senior Member, IEEE, Meltem Elitaş, and Kouhei Ohnishi, Fellow, IEEE
}

\begin{abstract}
In this paper, a sliding-mode-based design framework for fully actuated mechanical multibody system is discussed. The framework is based on the possibility to represent complex motion as a collection of tasks and to find effective mapping of the system coordinates that allows decoupling task and constraint control so one is able to enforce concurrently, or in certain time succession, the task and the constraints. The approach seems naturally encompassing the control of motion systems in interaction, and it allows application to bilateral control, multilateral control, etc. Such an approach leads to a more natural interpretation of the system tasks, simpler controller design, and easier establishment of the systems hierarchy. It allows a unified mathematical treatment of task control in the presence of constraints required to be satisfied by the system coordinates. In order to show the applicability of the proposed techniques, simulation and experimental results for high-precision systems in microsystem assembly tasks and bilateral control systems are presented.
\end{abstract}

Index Terms-Bilateral control, constrained multibody systems, force control, motion control, nonlinear systems, sliding-mode control (SMC).

\section{INTRODUCTION}

$\mathbf{M}$ OTION CONTROL systems are expected to be applied in unstructured environment where the presence of humans is natural. In many cases, such systems are acting as "agent" between skilled human operator and environment (surgery, micropart handling, teleoperation, etc.); thus, design of control should encompass a wide range of very demanding tasks. At the lower level, one should consider tasks of controlling individual systems. On the system level, control of bilateral or multilateral interaction between systems of the same or different nature, the remote control in master-slave systems, haptics, etc., should be considered. Such a complexity of motion control system poses a challenge for control systems designers.

Although design methods for decentralized control systems are interesting as concepts, a simple framework in view of

Manuscript received March 2, 2008; revised June 13, 2008. First published July 9, 2008; last published August 29, 2008 (projected). This work was supported in part by the TÜBITAK Project 106E040 and in part by a Grantin-Aid for the Global Center of Excellence for High-Level Global Cooperation for Leading-Edge Platform on Access Spaces from the Ministry of Education, Culture, Sport, Science, and Technology, Tokyo, Japan.

A. S̆abanović is with Sabanci University, Istanbul 34956, Turkey (e-mail: asif@sabanciuniv.edu).

M. Elitaş was with Sabanci University, Istanbul 34956, Turkey. She is now with the School of Life Sciences, École Polytechnique Fédérale de Lausanne, 1015 Lausanne, Switzerland (e-mail: meltem.elitas@epfl.ch).

K. Ohnishi is with Keio University, Yokohama 223-8522, Japan (e-mail: ohnishi@sd.keio.ac.jp).

Color versions of one or more of the figures in this paper are available online at http://ieeexplore.ieee.org.

Digital Object Identifier 10.1109/TIE.2008.928112 controller design is desired to cope with complexity of motion systems in interaction. In [1], it has been shown that under certain conditions, overall control input can be designed by linear superposition; in [2], a decoupled design method that makes a bilateral control system behave as a common passive rigid mechanical tool is proposed. In [3], a framework of controller design based on functionality is discussed; in [4], a bilateral control using sliding-mode control (SMC) applying functionality has been implemented. Basic approach in the control of bilateral system widely used in literature [5]-[7] is based on the design of controllers for master and slave side separately and then adding interacting terms in order to reach transparency requirements. Control of interconnected motion systems (bilateral and multilateral) in the framework of the acceleration control is discussed in [3] and [8]. All these works are based on the linearization of the individual systems by introducing the disturbance compensation in the joint space and then applying the acceleration control. This framework is shown to be very powerful, and it allows the application of multilateral systems and systems in interaction.

In this paper, a sliding-mode-based design framework for fully actuated mechanical multibody system is presented. The proposed approach leads to a more natural interpretation of the system tasks, simpler controller design, and easier establishment of the systems hierarchy. The possibility to interpret desired functional relation between one or more motion systems as a requirement that the system state is constrained in a manifold represents a basis of the proposed algorithm.

The application of SMC in motion control systems [9] ranges from control of power converters, electrical machines, robotic manipulators, mobile robots, PZT-based actuators, etc. The most salient feature of the SMC is a possibility to constrain system motion on the selected manifold in the state space; thus, this framework seems a natural candidate for the task that we are working toward in this paper-namely, maintaining selected functional relation between systems. In discrete time, this control that enforces sliding mode is continuous in the sense of the discrete-time systems, and the resulting intersampling motion for systems with smooth disturbances is constrained to the $o\left(T^{2}\right)$ vicinity of the sliding manifold [10], [11].

The organization of this paper is as follows. In Section II, the problem formulation and the general solution are discussed for $n$-degrees of freedom (DOF) fully actuated mechanical multibody system with and/or without motion modification due to interaction with environment. In Section III, the problems related to the task control in constrained systems are discussed, an SMC solution is proposed, and examples are shown in order to demonstrate the applicability of the proposed framework. 


\section{Control Problem Formulation}

For fully actuated mechanical system $S$, mathematical model may be found in the following form:

$$
\begin{aligned}
S: \mathbf{M}(\mathbf{q}) \ddot{\mathbf{q}}+\mathbf{b}(\mathbf{q}, \dot{\mathbf{q}})+\mathbf{g}(\mathbf{q}) & =\boldsymbol{\tau}-\boldsymbol{\tau}_{\mathrm{r}}\left(\mathbf{q}, \mathbf{q}_{\mathrm{e}}\right) \\
\mathbf{N}(\mathbf{q}, \dot{\mathbf{q}}) & =\mathbf{b}(\mathbf{q}, \dot{\mathbf{q}})+\mathbf{g}(\mathbf{q}) \\
\boldsymbol{\tau}_{\mathrm{r}}\left(\mathbf{q}, \mathbf{q}_{\mathrm{e}}\right) & = \begin{cases}\boldsymbol{\tau}_{\mathrm{r}}\left(\mathbf{q}, \mathbf{q}_{\mathrm{e}}\right), & \text { when in contact } \\
0, & \text { without contact }\end{cases}
\end{aligned}
$$

where $\mathbf{q}, \dot{\mathbf{q}} \in \Re^{n}$ stand for the vectors of generalized positions and generalized velocities, respectively, and $\mathbf{M}(\mathbf{q}) \in \Re^{n \times n}$ is the generalized positive definite inertia matrix with bounded parameters; hence, $M^{-} \leq\|\mathbf{M}(\mathbf{q})\| \leq M^{+} . \mathbf{N}(\mathbf{q}, \dot{\mathbf{q}}) \in \Re^{n \times 1}$ represents the vector of coupling forces, including gravity and friction, and is bounded by $\|\mathbf{N}(\mathbf{q}, \dot{\mathbf{q}})\| \leq N^{+} ; \boldsymbol{\tau} \in \Re^{n \times 1}$ with $\|\boldsymbol{\tau}\| \leq \tau_{0}$ is the vector of generalized input torques; $\boldsymbol{\tau}_{\mathrm{r}} \in \Re^{n \times 1}$ with $\left\|\boldsymbol{\tau}_{\mathrm{r}}\right\| \leq \tau_{\mathrm{rmt}}$ is the vector of interaction torques being zero when system $S$ is not interacting with the environment or other system; and $\mathbf{q}_{\mathrm{e}} \in \Re^{l}$ stands for the vector of generalized positions of environment. $M^{-}, M^{+}, N^{+}, \tau_{0}$, and $\tau_{\text {rmt }}$ are known scalars. Vectors $\boldsymbol{\tau}_{\mathrm{r}}$ and $\mathbf{N}(\mathbf{q}, \dot{\mathbf{q}})$ are assumed to satisfy matching conditions [12].

For the purpose of this paper, environment is treated as another mechanical system, and the interaction is represented by the mechanical force acting as a result of such an interaction. It is now obvious that such an external force can be treated as an additional input to the system (1) that is able to modify the system behavior in the same way as the control input does.

The configuration of the system can be represented by a single-valued vector function $\boldsymbol{\xi}(\mathbf{q}, \dot{\mathbf{q}}) \in \Re^{n \times 1}$. The control tasks for the system (1) may be represented as selected functions of the system configuration. The motion control can be formulated as the requirement to maintain the desired functional relation between the actual and the desired configurations on the trajectories of the system (1). This requirement can be interpreted as enforcing the state in the manifold

$$
\begin{aligned}
S_{q}=\{\mathbf{q}, \dot{\mathbf{q}}: & \left.\boldsymbol{\sigma}\left(\boldsymbol{\xi}(\mathbf{q}, \dot{\mathbf{q}}), \boldsymbol{\xi}^{\mathrm{ref}}\right)=\mathbf{0}\right\}, \\
& \boldsymbol{\sigma}, \boldsymbol{\xi}, \boldsymbol{\xi}^{\mathrm{ref}} \in \Re^{n \times 1} ; \quad \boldsymbol{\sigma}=\left[\sigma_{1}, \sigma_{2}, \ldots, \sigma_{n}\right]^{\mathrm{T}}
\end{aligned}
$$

where $\sigma \in \Re^{n \times 1}$ stands for the linear or nonlinear singlevalued vector function to be determined depending on the task of the overall system and the control system technical specification; $\boldsymbol{\xi}^{\text {ref }}(t) \in \Re^{n \times 1}$ stands for the reference configuration and is assumed to be a smooth bounded function with a continuous first-order time derivative. Requirement (2) can be satisfied if the solution $\boldsymbol{\sigma}\left(\boldsymbol{\xi}(\mathbf{q}, \dot{\mathbf{q}}), \boldsymbol{\xi}^{\mathrm{ref}}(t)\right)=\mathbf{0}$ is stable on the trajectories of system (1). With such a formulation, the controller design is related to ensuring the stability of $\boldsymbol{\sigma}\left(\boldsymbol{\xi}(\mathbf{q}, \dot{\mathbf{q}}), \boldsymbol{\xi}^{\mathrm{ref}}(t)\right)=\mathbf{0}$. The question of the definition of the operational tasks of system (1) in terms of the system configuration and the admissible structure of the desired functional relation is still open and will require careful examination in order to complete the overall control design. This problem will be addressed later in this paper.

\section{A. Equations of Motion and Control Input Selection}

The motion of the system if constraint (2) is satisfied, and the selection of the control to enforce the stability of solution $\boldsymbol{\sigma}\left(\boldsymbol{\xi}(\mathbf{q}, \dot{\mathbf{q}}), \boldsymbol{\xi}^{\mathrm{ref}}(t)\right)=\mathbf{0}$ should be discussed first. Equations of motion can be found by using the so-called equivalent control method [10]. In this method, the control input is taken as the solution of $\left.\boldsymbol{\sigma}\left(\boldsymbol{\xi}(\mathbf{q}, \dot{\mathbf{q}}), \boldsymbol{\xi}^{\mathrm{ref}}(t)\right)\right|_{\tau=\tau_{\text {eq }}}=\mathbf{0}$. This solution can be derived as

$$
\begin{aligned}
\boldsymbol{\tau}_{\mathrm{eq}}=\left(\boldsymbol{\tau}_{\mathrm{r}}\left(\mathbf{q}, \mathbf{q}_{\mathrm{e}}\right)+\mathbf{N}\right. & (\mathbf{q}, \dot{\mathbf{q}})) \\
& -\left(\mathbf{Q M}^{-1}\right)^{-1}\left(\mathbf{H} \dot{\boldsymbol{\xi}}^{\mathrm{ref}}(t)+\mathbf{C} \dot{\mathbf{q}}\right) .
\end{aligned}
$$

Matrices $\quad \mathbf{C}=[\partial \sigma / \partial \xi][\partial \xi / \partial q], \quad Q=[\partial \sigma / \partial \xi][\partial \xi / \partial \dot{q}], \quad$ and $H=\left[\partial \sigma / \partial \xi^{\text {ref }}\right]$ are assumed to have a full rank for $\forall(\mathbf{q}, \dot{\mathbf{q}}) \in$ $S_{q}$. The relation $\left(\mathbf{Q M}^{-1}\right)^{-1}=\mathbf{M Q}^{-1}$ is true due to the properties of the inertia matrix. By inserting (3) into (1), the equations of motion of system (1) in manifold (2) are obtained in the following form:

$$
\begin{aligned}
\mathbf{M} \ddot{\mathbf{q}} & =-\left(\mathbf{Q} \mathbf{M}^{-1}\right)^{-1}\left(\mathbf{H} \dot{\boldsymbol{\xi}}^{\text {ref }}(t)+\mathbf{C} \dot{\mathbf{q}}\right) \\
& =\mathbf{M} \ddot{\mathbf{q}}^{\text {des }} \Rightarrow \ddot{\mathbf{q}}=\ddot{\mathbf{q}}^{\text {des }} \\
\ddot{\mathbf{q}}^{\text {des }} & =-\mathbf{Q}^{-1}\left(\mathbf{H} \dot{\boldsymbol{\xi}}^{\text {ref }}(t)+\mathbf{C} \dot{\mathbf{q}}\right) .
\end{aligned}
$$

Motion (4) is equivalent to the acceleration control [13] with desired acceleration $\ddot{\mathbf{q}}^{\mathrm{des}}=-\mathbf{Q}^{-1}\left(\mathbf{H} \dot{\boldsymbol{\xi}}^{\text {ref }}(t)+\mathbf{C} \dot{\mathbf{q}}\right)$ and is valid from the time $t \geq t_{0}$ with $t_{0}$ being the moment that the state of the system reaches manifold (2). If closed-loop motion (4) should be modified due to the interaction with other systems or environment, the desired acceleration $\ddot{\mathbf{q}}^{\text {des }}$ must depend on the interaction force.

Control can be selected by selecting Lyapunov function candidate $\nu=(1 / 2) \boldsymbol{\sigma}^{\mathrm{T}} \boldsymbol{\sigma}>0 \quad \nu(0)=0$ and enforcing its derivative to be $\dot{\nu}=\boldsymbol{\sigma}^{\mathrm{T}} \dot{\boldsymbol{\sigma}}=-\boldsymbol{\sigma}^{\mathrm{T}} \mathbf{\Psi}(\boldsymbol{\sigma})<0$. If $-\boldsymbol{\sigma}^{\mathrm{T}} \mathbf{\Psi}(\boldsymbol{\sigma})=$ $-\rho \nu^{\delta}<0, \rho>0$, and $(1 / 2) \leq \delta<1$ [14], the manifold (2) will be attractive, and the stability conditions for solution $\boldsymbol{\sigma}\left(\boldsymbol{\xi}, \boldsymbol{\xi}^{\text {ref }}\right)=\mathbf{0}_{n \times 1}$ are satisfied. From $\dot{\nu}=\boldsymbol{\sigma}^{\mathrm{T}} \dot{\boldsymbol{\sigma}}=-\boldsymbol{\sigma}^{\mathrm{T}} \boldsymbol{\Psi}(\boldsymbol{\sigma})$, one can derive $\boldsymbol{\sigma}^{\mathrm{T}}(\dot{\boldsymbol{\sigma}}+\boldsymbol{\Psi}(\boldsymbol{\sigma}))=0$, and consequently, nontrivial solution can be obtained as

$$
\boldsymbol{\tau}=\boldsymbol{\tau}_{\text {eq }}-\left(\mathbf{Q M}^{-1}\right)^{-1} \mathbf{\Psi}(\boldsymbol{\sigma})=\boldsymbol{\tau}_{\text {eq }}-\mathrm{MQ}^{-1} \mathbf{\Psi}(\boldsymbol{\sigma}) .
$$

For continuous-time system function, $\boldsymbol{\Psi}(\boldsymbol{\sigma})$ is most often selected as $-\boldsymbol{\sigma}^{\mathrm{T}} \boldsymbol{\Psi}(\boldsymbol{\sigma})=-\rho \nu^{1 / 2}$. Being discontinuous, such a control input may cause chattering in mechanical systems. For real system, chattering may be a problem, and many possibilities to avoid or minimize chattering in mechanical systems are presented in [15]. In the real system, control is bounded $\|\boldsymbol{\tau}\| \leq \tau_{0}$; thus, (5) should be modified to take this into account

$$
\boldsymbol{\tau}=\operatorname{sat}\left(\boldsymbol{\tau}_{\text {eq }}-\mathbf{M Q}^{-1} \mathbf{\Psi}(\boldsymbol{\sigma})\right)
$$

where $\operatorname{sat}(\bullet)$ stands for the saturation function with bounds $\tau_{b}=\tau_{0} \boldsymbol{\tau} /\|\boldsymbol{\tau}\|$. The implementation of the control (6) requires full knowledge of the plant parameters and disturbances. An approximate solution can be found by estimating equivalent control as $\hat{\boldsymbol{\tau}}_{\mathrm{eq}}=\left(\boldsymbol{\tau}-\mathbf{M Q}^{-1} \boldsymbol{s} \boldsymbol{\sigma}\right) g /(s+g)$ and replacing $\boldsymbol{\tau}_{\mathrm{eq}}$ 
in (6). In this case, the control will enforce relation $\dot{\sigma}+$ $\boldsymbol{\Psi}(\boldsymbol{\sigma})=\mathbf{p}_{\text {eq }}=\boldsymbol{\tau}_{\text {eq }}-\hat{\boldsymbol{\tau}}_{\text {eq }}$. This approximation will introduce bounded error since $g \rightarrow \infty \Rightarrow \hat{\tau}_{\text {eq }} \rightarrow \tau_{\text {eq }}$. If (3) is inserted in (6) and the disturbance observer [13] is applied, the control becomes

$$
\boldsymbol{\tau}=\operatorname{sat}\left(\left(\hat{\boldsymbol{\tau}}_{\mathrm{r}}+\hat{\mathbf{N}}\right)-\mathbf{M} \mathbf{Q}^{-1}\left(\mathbf{H} \dot{\boldsymbol{\xi}}^{\mathrm{ref}}(t)+\mathbf{C} \dot{\mathbf{q}}+\mathbf{\Psi}(\boldsymbol{\sigma})\right)\right)
$$

Approximated control (7) enforces the relation $\dot{\boldsymbol{\sigma}}+\boldsymbol{\Psi}(\boldsymbol{\sigma})=$ $\mathbf{p}, \mathbf{p}=\left(\boldsymbol{\tau}_{\mathrm{r}}+\mathbf{N}\right)-\left(\hat{\boldsymbol{\tau}}_{\mathrm{r}}+\hat{\mathbf{N}}\right)$, and as in the previous case, error is bounded if observer error is bounded. Control (7) represents a generic acceleration controller enforcing the attractiveness and the stability of solution $\boldsymbol{\sigma}\left(\boldsymbol{\xi}, \boldsymbol{\xi}^{\text {ref }}\right)=\mathbf{0}$.

In the discrete time with sampling interval " $T$ " with $\dot{\boldsymbol{\sigma}}(k-$ $1)=(\boldsymbol{\sigma}(k)-\boldsymbol{\sigma}(k-1)) / T$ and due to the fact that equivalent control is continuous, one can write

$$
\boldsymbol{\tau}(k) \cong \operatorname{sat}\left(\boldsymbol{\tau}_{\text {eq }}(k-1)-\mathbf{M Q}^{-1} \boldsymbol{\Psi}\left(\boldsymbol{\sigma}_{k}\right)\right)
$$

The error introduced by this approximation is on the order of $o\left(T^{2}\right)$ [16]. In general, the thickness of the error layer can be determined by evaluating

$$
\boldsymbol{\sigma}(k T+\tau)-\boldsymbol{\sigma}(k T)=-\int_{k T}^{k T+\tau} \boldsymbol{\Psi}(\boldsymbol{\sigma}(t)) \mathrm{d} t+o\left(T^{2}\right)
$$

With $\boldsymbol{\Psi}(\boldsymbol{\sigma}(t))$ being proportional to $\boldsymbol{\sigma}(t)$, the thickness of the boundary layer is on the order of $o\left(T^{2}\right)$. If relay control is applied, it will result in motion with chattering within a boundary layer having a thickness on the order of $o(T)$.

\section{B. Selection of Reference Configuration}

Without loss of generality, constant matrices $\mathbf{C}, \mathbf{Q}$, and $\mathbf{D}$ can be defined; thus, the system configuration and its reference become $\boldsymbol{\xi}_{q}=\mathbf{Q} \dot{\mathbf{q}}+\mathbf{C q}$ and $\boldsymbol{\xi}_{q}^{\text {ref }}=\mathbf{Q} \dot{\mathbf{q}}^{\text {ref }}+\mathbf{C q}^{\text {ref }}$. Consequently, $S_{q}=\left\{\mathbf{q}, \dot{\mathbf{q}}: \boldsymbol{\sigma}\left(\boldsymbol{\xi}_{q}, \boldsymbol{\xi}_{q}^{\text {ref }}\right)=\boldsymbol{\xi}_{q}^{\text {ref }}-\boldsymbol{\xi}_{q}=0\right\}$ stays for the constraint manifold. The control (7) or (8) can be directly applied. If one selects $\Psi\left(\boldsymbol{\sigma}_{q}\right)=-\mathbf{D} \boldsymbol{\sigma}_{q} ; \mathbf{D}>0$, the equations of motion become

$$
\mathrm{Q} \Delta \ddot{\mathrm{q}}+(\mathrm{C}+\mathrm{DQ}) \Delta \dot{\mathrm{q}}+\mathrm{CQ} \Delta \mathrm{Q}=\mathbf{0}, \quad \Delta \mathrm{q}=\mathrm{q}-\mathrm{q}^{\mathrm{ref}}
$$

For $\mathbf{Q}=\mathbf{I}$, (10) represents unit mass systems - the same as the one obtained by application of the disturbance observer and the PD controller [13].

If the reaction torque can be modeled as $\tau_{\mathrm{r}}=\mathbf{K}_{\mathrm{P}} \boldsymbol{\Delta} \mathbf{q}_{\mathrm{e}}+$ $\mathbf{K}_{\mathrm{D}} \boldsymbol{\Delta} \dot{\mathbf{q}}_{\mathrm{e}}$, with $\Delta \mathbf{q}_{\mathrm{e}}=\mathbf{q}-\mathbf{q}_{\mathrm{e}}$ and $\mathbf{K}_{\mathrm{P}}, \mathbf{K}_{\mathrm{D}}$ diagonal matrices of appropriate dimensions, then with $\boldsymbol{\xi}_{F}^{\text {ref }}=\boldsymbol{\tau}^{\text {ref }}+\left(\mathbf{K}_{\mathrm{P}} \mathbf{q}_{\mathrm{e}}+\right.$ $\left.\mathbf{K}_{\mathrm{D}} \dot{\mathbf{q}}_{\mathrm{e}}\right)$, the sliding mode manifold $S_{F}=\left\{\mathbf{q}, \dot{\mathbf{q}}:\left(\mathbf{K}_{\mathrm{P}} \mathbf{q}+\right.\right.$ $\left.\left.\mathbf{K}_{\mathrm{D}} \dot{\mathbf{q}}\right)-\boldsymbol{\xi}_{F}^{\mathrm{ref}}=\boldsymbol{\sigma}_{F}=0\right\}$ has the same form as the one derived for the trajectory tracking; thus, the structure of the control input is obtained as $\boldsymbol{\tau}=\boldsymbol{\tau}_{\text {eq }}-\mathbf{M K}_{\mathrm{D}}^{-1} \boldsymbol{\Psi}\left(\boldsymbol{\sigma}_{F}\right)$.

\section{Motion Modification by Interaction Forces}

Assume two mechanical systems $S_{i}$ and $S_{j}$

$$
\begin{aligned}
S_{i}: \mathbf{M}_{i}\left(\mathbf{q}_{i}\right) \ddot{\mathbf{q}}_{i}+\mathbf{N}_{i}\left(\mathbf{q}_{i}, \dot{\mathbf{q}}_{i}\right) & =\boldsymbol{\tau}_{i}-\mathbf{g}_{i j}\left(\mathbf{q}_{i}, \mathbf{q}_{j}\right) \\
S_{j}: \mathbf{M}_{j}\left(\mathbf{q}_{j}\right) \ddot{\mathbf{q}}_{j}+\mathbf{N}_{j}\left(\mathbf{q}_{j}, \dot{\mathbf{q}}_{j}\right) & =\boldsymbol{\tau}_{j}+\mathbf{g}_{i j}\left(\mathbf{q}_{i}, \mathbf{q}_{j}\right) .
\end{aligned}
$$

Assume reference configuration $\boldsymbol{\xi}_{i q}^{\mathrm{ref}}(t)$ and $\boldsymbol{\xi}_{j q}^{\mathrm{ref}}(t)$, respectively. Interaction force between systems $S_{i}$ and $S_{j}$ is $\mathbf{g}_{i j} \in$ $\Re^{n \times 1}$, and $\mathbf{g}_{i j}=\mathbf{0}$ if systems are not interacting. Let $\mathbf{g}_{i j}$ be modeled as spring damper $\mathbf{g}_{i j}=\mathbf{K}_{\mathrm{P} i} \Delta \mathbf{q}+\mathbf{K}_{\mathrm{D} i} \Delta \dot{\mathbf{q}}, \Delta \mathbf{q}=$ $\mathbf{q}_{i}-\mathbf{q}_{j}$, and $\mathbf{g}_{i j}^{\text {ref }}(t)$ being the desired value while the systems are in interaction. Assume that system $S_{j}$ is controlled in the trajectory tracking mode. Then, in order to maintain the desired profile of the interaction force, system $S_{i}$ should change its configuration as a result of the interaction.

The design of the control will follow the same steps as for the position tracking system, but, as shown in (4), the reference configuration must include both the trajectory tracking and the interaction control. Let us select the sliding mode manifold in the following form:

$$
S_{i q}=\left\{\mathbf{q}_{i}, \dot{\mathbf{q}}_{i}: \mathbf{C}_{i} \mathbf{q}_{i}+\mathbf{Q}_{i} \dot{\mathbf{q}}_{i}-\boldsymbol{\xi}_{i q F}^{\mathrm{ref}}(t)=\boldsymbol{\sigma}_{i q F}=\mathbf{0}\right\}
$$

The reference configuration is selected the same way as for the trajectory tracking with an additional term that depends on the way that the system is required to react on the interaction with environment. The following structures can be taken as examples:

1) $\boldsymbol{\xi}_{i q F}^{\mathrm{ref}}(t)=\boldsymbol{\xi}_{i q}^{\mathrm{ref}}(t)-\boldsymbol{\Gamma g}_{i j}\left(\mathbf{q}_{i}, \mathbf{q}_{j}\right)$ (compliant motion);

2) $\boldsymbol{\xi}_{i q F}^{\mathrm{ref}}(t)=\boldsymbol{\xi}_{i q}^{\mathrm{ref}}(t)-\vartheta\left(\mathbf{g}_{i j}^{\mathrm{ref}}(t), \mathbf{g}_{i j}\left(\mathbf{q}_{i}, \mathbf{q}_{j}\right)\right)$ (force tracking);

3) $\boldsymbol{\xi}_{i q F}^{\mathrm{ref}}(t)=\boldsymbol{\xi}_{i q}^{\mathrm{ref}}(t)-\left(\vartheta\left(\mathbf{g}_{i j}^{\mathrm{ref}}(t), \mathbf{g}_{i j}\left(\mathbf{q}_{i}, \mathbf{q}_{j}\right)\right)+\right.$ $\left.\boldsymbol{\Gamma g}_{i j}\left(\mathbf{q}_{i}, \mathbf{q}_{j}\right)\right)$ [the combination of cases 1) and 2)].

Matrix $\Gamma$ is a diagonal compliance matrix with elements different from zero in the directions in which compliance is to be maintained, and zero in the directions in which either contact force or trajectory tracking should be maintained. The output of the force tracking controller enforces sliding mode in $S_{i j F}=\left\{\mathbf{q}_{i}, \dot{\mathbf{q}}_{i}: \mathbf{K}_{\mathrm{P} i} \mathbf{q}_{i}+\mathbf{K}_{\mathrm{D} i} \dot{\mathbf{q}}_{i}-\boldsymbol{\xi}_{F}^{\mathrm{ref}}=\boldsymbol{\sigma}_{F}=\mathbf{0}\right\}$ with the reference $\boldsymbol{\xi}_{F}^{\text {ref }}=\left(\mathbf{g}_{i j}^{\text {ref }}+\mathbf{K}_{\mathrm{P} i} \mathbf{q}_{j}+\mathbf{K}_{\mathrm{D} i} \dot{\mathbf{q}}_{j}\right)$. The force control input is selected as $\vartheta=\vartheta_{\text {eq }}-\mathbf{M}_{i} \mathbf{K}_{\mathrm{D} i}^{-1} \boldsymbol{\Psi}\left(\boldsymbol{\sigma}_{F i j}\right)$ if systems are interacting and if they are not $\vartheta=\mathbf{0}$. Control input that enforces sliding mode in manifold (12) is as defined in (5) $\tau_{i}=\tau_{\text {eq } i}$ $\mathbf{M}_{i} \mathbf{Q}_{i}^{-1} \boldsymbol{\Psi}\left(\boldsymbol{\sigma}_{i q F}\right)$ via appropriate changes of variables.

\section{Example}

Experimental verification is performed on the setup consisting of Piezomechanik's PSt150/5/60 stack actuator $\left(x_{\max }=\right.$ $60 \mu \mathrm{m}, F_{\max }=800 \mathrm{~N}$, and $\nu_{\max }=150 \mathrm{~V}$ ) connected to SVR150/3 low-voltage low-power amplifier; force measurement is realized by a load cell. Control is implemented in the dSPACE DS1103 module hosted in a PC. The aim is to demonstrate the modification of motion due to the interaction with environment. Free motion, contact with environment, and control of the contact force are shown in Fig. 1. $x_{\mathrm{r}}=20+$ $\cos (0.5 t)$ stands for the position reference in micrometers. 

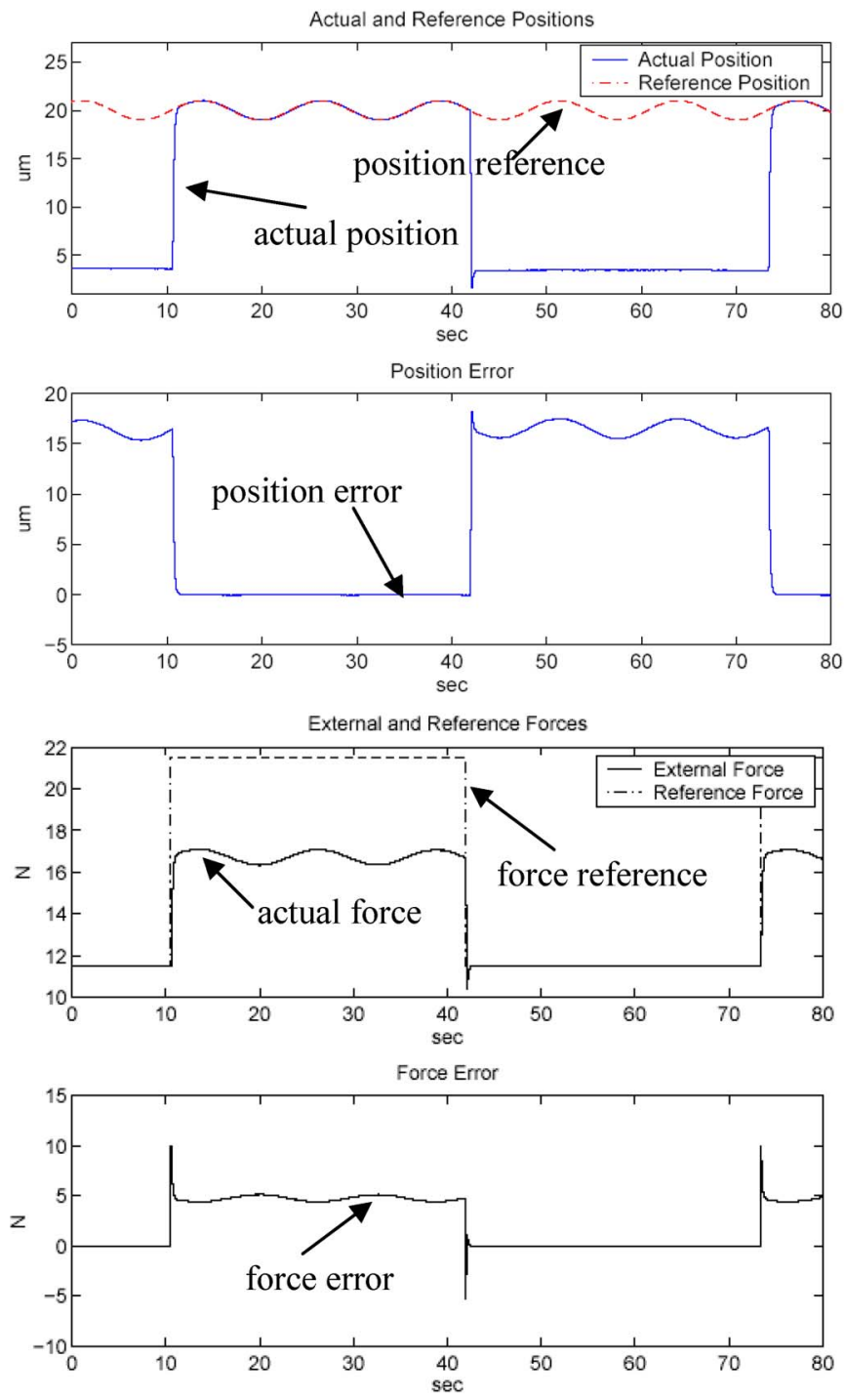

Fig. 1. Experimental behavior of the PZT actuator with SMC control upper half depicting the transients in position and lower half depicting the transients in force control.

The force modifies the trajectory if $F_{\mathrm{r}}=11.5[\mathrm{~N}]$. The higher value of the reference force $F_{\mathrm{r}}=22.5[\mathrm{~N}]$ is set such that it does not interfere with position tracking. The sliding-mode manifold (12) is selected as $\sigma=C \Delta x+\Delta \dot{x}-\alpha \vartheta_{F}, C=800$, and $\alpha=0$ when there is no interaction $\alpha=1$ in opposite case. Control is selected as in (6) with $\Psi=-D \sigma$ and $D=2500$. The force control is $\vartheta_{F}=\hat{\vartheta}_{F \text { eq }}-\eta_{F} D_{F} \sigma_{F}, \sigma_{F}=F_{\mathrm{e}}-F_{\mathrm{r}}, D_{F}=$ 1900 , and $\eta_{F}=0.25$. The transitions from position tracking to force tracking and vice versa are clearly shown in figures-the position tracking error is large when force is tracking and vice versa.

\section{Multibody Systems in Interaction}

\section{A. Physically Constrained Systems}

For motion control systems, of particular interest is to maintain the desired functional relation between subsystems by acting on all of the subsystems. Assume a set of motion systems, each described by $S_{i}: \mathbf{m}_{i}\left(\mathbf{q}_{i}\right) \ddot{\mathbf{q}}_{i}+\mathbf{b}_{i}\left(\mathbf{q}_{i}, \dot{\mathbf{q}}_{i}\right)+\mathbf{g}_{i}(\mathbf{q})=\boldsymbol{\tau}_{i}$, $i=1,2, \ldots, n$. The motion of the overall system can be described in the configuration space by the following compound model:

$$
S: \mathbf{M}(\mathbf{q}) \ddot{\mathbf{q}}+\mathbf{b}(\mathbf{q}, \dot{\mathbf{q}})+\mathbf{g}(\mathbf{q})=\boldsymbol{\tau}
$$

where $\mathbf{q}, \boldsymbol{\tau}, \mathbf{b}$, and $\mathbf{g}$ are $n \times 1$ vectors, and $\mathbf{M}$ is $n \times n$ full rank inertia matrix.

Assume that subsystems are physically interconnected to satisfy the set of $m$ constraints $\phi(\mathbf{q})=\mathbf{0}$ with $m \times n$ constraint matrix $\partial \phi(\mathbf{q}) / \partial \mathbf{q}=\mathbf{J}_{\varphi}$. The interaction torque can be expressed as $\boldsymbol{\tau}_{i j}=\mathbf{J}_{\varphi}^{\mathrm{T}} \boldsymbol{\lambda}$, and (13) becomes

$$
S: \mathbf{M}(\mathbf{q}) \ddot{\mathbf{q}}+\mathbf{b}(\mathbf{q}, \dot{\mathbf{q}})+\mathbf{g}(\mathbf{q})=\boldsymbol{\tau}+\mathbf{J}_{\varphi}^{\mathrm{T}} \boldsymbol{\lambda}
$$

where $\boldsymbol{\lambda}$ is a vector of unknown Lagrange multipliers. The dynamics (14) can be determined in many ways. Here, we will formally apply the ideas of sliding modes. Since the constraint equations require $\dot{\phi}(\mathbf{q})=\mathbf{J}_{\varphi} \dot{\mathbf{q}}=\mathbf{0}$, the value of $\boldsymbol{\lambda}$ can be determined such that sliding mode is enforced in $\dot{\phi}(\mathbf{q})=\mathbf{0}$. From $\ddot{\phi}\left(\mathbf{q}, \boldsymbol{\lambda}=\boldsymbol{\lambda}_{\mathrm{eq}}\right)=\mathbf{0}$, one can find

$$
\begin{aligned}
\boldsymbol{\lambda}_{\mathrm{eq}}=-\left(\mathbf{J}_{\varphi} \mathbf{M}^{-1} \mathbf{J}_{\varphi}^{\mathrm{T}}\right)^{-1} \mathbf{J}_{\varphi} \mathbf{M}^{-1}(\boldsymbol{\tau}-\mathbf{b}-\mathbf{g}) & \\
& +\mathbf{J}_{\varphi}^{\mathrm{T}}\left(\mathbf{J}_{\varphi} \mathbf{M}^{-1} \mathbf{J}_{\varphi}^{\mathrm{T}}\right)^{-1} \dot{\mathbf{J}}_{\varphi} \dot{\mathbf{q}} .
\end{aligned}
$$

By substituting (15) into (14), the motion of the constrained system becomes

$$
\mathbf{M} \ddot{\mathbf{q}}+\mathbf{b}+\mathbf{g}-\mathbf{J}_{\varphi}^{\mathrm{T}}\left(\tilde{\mathbf{J}}_{\varphi}^{\mathrm{T}}(\mathbf{b}+\mathbf{g})+\left(\mathbf{J}_{\varphi} \mathbf{M}^{-1} \mathbf{J}_{\varphi}^{\mathrm{T}}\right)^{-1} \dot{\mathbf{J}}_{\varphi} \dot{\mathbf{q}}\right)=\mathbf{N}_{\phi}^{\mathrm{T}} \boldsymbol{\tau}
$$

$\tilde{\mathbf{J}}_{\varphi}^{\mathrm{T}}=\left(\mathbf{J}_{\varphi} \mathbf{M}^{-1} \mathbf{J}_{\varphi}^{\mathrm{T}}\right)^{-1} \mathbf{J}_{\varphi} \mathbf{M}^{-1}$ stand for the transpose of mass weighted right pseudo inverse and the transpose of the null space matrix $\mathbf{N}_{\varphi}^{\mathrm{T}}=\left(\mathbf{I}-\mathbf{J}_{\varphi}^{\mathrm{T}} \tilde{\mathbf{J}}_{\varphi}^{\mathrm{T}}\right)$. Equation (16), along with constraint $\phi(\mathbf{q})=\mathbf{0}$, describes the motion of $(n-m)$ order dynamic system. The component $\mathbf{N}_{\varphi}^{\mathrm{T}} \boldsymbol{\tau}$ of the generalized torque can be assigned to the realization of other tasks of the system.

\section{B. Virtually Constrained Systems}

Let us now analyze the possibility to control the system (13) that enforces a set of $m$ virtual constraints $\phi(\mathbf{q})=\mathbf{0}$ with constraint matrix $[\partial \phi(\mathbf{q}) / \partial \mathbf{q}]=\mathbf{J}_{\varphi}$. For this reason, sliding mode can be enforced on the following manifold:

$$
S_{\varphi}=\left\{\mathbf{q}, \dot{\mathbf{q}}: \boldsymbol{\sigma}_{\varphi}\left(\boldsymbol{\xi}_{\varphi}(\boldsymbol{\phi}, \dot{\boldsymbol{\phi}}), \boldsymbol{\xi}_{\varphi}^{\mathrm{ref}}\right)=\mathbf{0}\right\} .
$$

$\boldsymbol{\xi}_{\varphi}(\boldsymbol{\phi}, \dot{\boldsymbol{\phi}})$ stands for $(m \times 1)$ configuration vector with $\boldsymbol{\xi}_{\varphi}^{\mathrm{ref}}$ as its reference. Without loss of generality, let $\boldsymbol{\sigma}_{\varphi}=\boldsymbol{\xi}_{\varphi}-\boldsymbol{\xi}_{\varphi}^{\mathrm{ref}}$, $\boldsymbol{\xi}_{\varphi}(\boldsymbol{\phi}, \dot{\boldsymbol{\phi}})=\mathbf{G}_{\varphi} \boldsymbol{\phi}+\dot{\boldsymbol{\phi}}, \mathbf{G}_{\varphi}>0, \operatorname{rank}\left(\mathbf{G}_{\varphi}\right)=m$, and $\boldsymbol{\xi}_{\varphi}^{\mathrm{ref}}=$ $\mathbf{0}$. The derivative $\dot{\boldsymbol{\sigma}}_{\varphi}$ can be expressed as

$$
\dot{\boldsymbol{\sigma}}_{\varphi}=\mathbf{J}_{\varphi} \mathbf{M}^{-1}(\boldsymbol{\tau}-\mathbf{b}-\mathbf{g})+\left(\mathbf{G}_{\varphi} \mathbf{J}_{\varphi}+\dot{\mathbf{J}}_{\varphi}\right) \dot{\mathbf{q}}
$$

As discussed in Section III-A, the torque can be expressed as $\boldsymbol{\tau}=\mathbf{N}_{\varphi}^{\mathbf{T}} \boldsymbol{\tau}_{0}+\mathbf{J}_{\varphi}^{\mathrm{T}} \mathbf{f}_{\varphi}$ where the second component should be 
selected to maintain system constraints. By substituting $\tau=$ $\mathbf{N}_{\varphi}^{\mathbf{T}} \boldsymbol{\tau}_{0}+\mathbf{J}_{\varphi}^{\mathrm{T}} \mathbf{f}_{\varphi}$ into (18), one can derive

$$
\dot{\boldsymbol{\sigma}}_{\varphi}=\mathbf{J}_{\varphi} \mathbf{M}^{-\mathbf{1}} \mathbf{J}_{\varphi}^{\mathrm{T}} \mathbf{f}_{\varphi}-\mathbf{J}_{\varphi} \mathbf{M}^{-\mathbf{1}}(\mathbf{b}+\mathbf{g})+\left(\mathbf{G}_{\varphi} \mathbf{J}_{\varphi}+\dot{\mathbf{J}}_{\varphi}\right) \dot{\mathbf{q}} .
$$

Let us rewrite (19) in the form

$$
\begin{aligned}
& \dot{\boldsymbol{\sigma}}_{\varphi}=\mathbf{f}_{\varphi}^{*}+\mathbf{d}_{\varphi}, \quad \mathbf{f}_{\varphi}^{*}=\mathbf{J}_{\varphi} \mathbf{M}^{-\mathbf{1}} \mathbf{J}_{\varphi}^{\mathrm{T}} \mathbf{f} \\
& \mathbf{d}_{\varphi}=-\mathbf{J}_{\varphi} \mathbf{M}^{-\mathbf{1}}(\mathbf{b}+\mathbf{g})+\left(\mathbf{G}_{\varphi} \mathbf{J}_{\varphi}+\dot{\mathbf{J}}_{\varphi}\right) \dot{\mathbf{q}}
\end{aligned}
$$

The exponential stability will be enforced if transients satisfy $\dot{\boldsymbol{\sigma}}_{\varphi}=-\mathbf{D}_{\varphi} \boldsymbol{\sigma}_{\varphi}, \mathbf{D}_{\varphi}>0$ and, thus, if control is selected as

$$
\mathbf{f}_{\varphi}^{*}=-\mathbf{d}_{\varphi}-\mathbf{D}_{\varphi} \boldsymbol{\sigma}_{\varphi} .
$$

By replacing real disturbance in (21) by its estimated value, the closed-loop dynamics can be expressed as

$$
\begin{aligned}
\dot{\boldsymbol{\sigma}}_{\varphi}+\mathbf{D}_{\varphi} \boldsymbol{\sigma}_{\varphi} & =\ddot{\boldsymbol{\phi}}+\left(\mathbf{G}_{\varphi}+\mathbf{D}_{\varphi}\right) \dot{\boldsymbol{\phi}}+\mathbf{G}_{\varphi} \mathbf{D}_{\varphi} \boldsymbol{\phi} \\
& =\mathbf{p}_{\varphi}=\mathbf{d}_{\varphi}-\hat{\mathbf{d}}_{\varphi} .
\end{aligned}
$$

The error due to the disturbance estimation $\left(\mathbf{d}_{\varphi}-\hat{\mathbf{d}}_{\varphi}\right)$ will be bounded if the estimation error is bounded. From $\mathbf{f}_{\varphi}^{*}=$ $-\hat{\mathbf{d}}_{\varphi}-\mathbf{D}_{\varphi} \boldsymbol{\sigma}_{\varphi}$ and $\mathbf{f}_{\varphi}^{*}=\mathbf{J}_{\varphi} \mathbf{M}^{-1} \mathbf{J}_{\varphi}^{\mathrm{T}} \mathbf{f}_{\varphi}$, the control torque can be calculated as

$$
\mathbf{J}_{\varphi}^{\mathrm{T}} \mathbf{f}_{\varphi}=-\mathbf{J}_{\varphi}^{\mathrm{T}}\left(\mathbf{J}_{\varphi} \mathbf{M}^{-\mathbf{1}} \mathbf{J}_{\varphi}^{\mathrm{T}}\right)^{-1}\left(\hat{\mathbf{d}}_{\varphi}+\mathbf{D}_{\varphi} \boldsymbol{\sigma}_{\varphi}\right) .
$$

By inserting $\boldsymbol{\tau}=\mathbf{N}_{\varphi}^{\mathbf{T}} \boldsymbol{\tau}_{0}+\mathbf{J}_{\varphi}^{\mathrm{T}} \mathbf{f}_{\varphi}$ and (23) into (13), the unconstrained motion of the system can be determined as

$$
\begin{aligned}
\mathbf{M} \ddot{\mathbf{q}}+\mathbf{b}+\mathbf{g}= & \mathbf{N}_{\varphi}^{\mathrm{T}} \boldsymbol{\tau}_{0}+\mathbf{J}_{\varphi}^{\mathrm{T}}\left(\mathbf{J}_{\varphi} \mathbf{M}^{-1} \mathbf{J}_{\varphi}^{\mathrm{T}}\right)^{-1} \mathbf{J}_{\varphi} \mathbf{M}^{-1}(\mathbf{b}+\mathbf{g}) \\
& -\mathbf{J}_{\varphi}^{\mathrm{T}}\left(\mathbf{J}_{\varphi} \mathbf{M}^{-1} \mathbf{J}_{\varphi}^{\mathrm{T}}\right)^{-1} \\
& \times\left(\left(\mathbf{G}_{\varphi} \mathbf{J}_{\varphi}+\dot{\mathbf{J}}_{\varphi}\right) \dot{\mathbf{q}}+\mathbf{D}_{\varphi} \boldsymbol{\sigma}_{\varphi}\right)
\end{aligned}
$$

This motion is very similar to (16). The difference is related to the enforcement of the transient (22). The component $\mathbf{N}_{\varphi}^{\mathrm{T}} \boldsymbol{\tau}_{0}$ could be synthesized to impose desired behavior of the constrained system as long as this behavior is not in conflict with the constraints.

\section{Task Control}

Consider a problem of designing control for system (13) such that the task vector $\mathbf{x}_{T}^{\mathrm{T}}=\left[\begin{array}{llll}x_{1}(\mathbf{q}) & \ldots & x_{k}(\mathbf{q})\end{array}\right]$ tracks its smooth reference $\mathbf{x}_{T}^{\text {ref }}$. Similar to the case of enforcing virtual constraint discussed in previous section, the task control involves finding a control that guarantees the sliding-mode existence in manifold

$$
S_{x T}=\left\{\mathbf{q}, \dot{\mathbf{q}}: \boldsymbol{\sigma}_{x T}\left(\boldsymbol{\xi}_{x T}\left(\mathbf{x}_{T}, \dot{\mathbf{x}}_{T}\right), \boldsymbol{\xi}_{x T}^{\mathrm{ref}}\right)=\mathbf{0}\right\}
$$

where $\boldsymbol{\xi}_{x T}\left(\mathbf{x}_{T} \dot{\mathbf{x}}_{T}\right), \boldsymbol{\xi}_{x T}^{\text {ref }}$ stand for $(k \times 1)$ configuration vector and its reference, respectively. Let $\boldsymbol{\xi}_{x T}=\mathbf{G}_{x T} \mathbf{x}_{T}+\dot{\mathbf{x}}_{T}$, $\mathbf{G}_{x T}>0, \operatorname{rank} \mathbf{G}_{x T}=k, \boldsymbol{\xi}_{x T}^{\text {ref }}=\mathbf{G}_{\varphi} \mathbf{x}_{T}^{\text {ref }}+\dot{\mathbf{x}}_{T}^{\text {ref }}$, and $\boldsymbol{\sigma}_{x T}=$ $\boldsymbol{\xi}_{x T}-\boldsymbol{\xi}_{x T}^{\text {ref }}$. The time derivative of $\boldsymbol{\sigma}_{x T}$ with $\mathbf{J}_{x T}=\partial \mathbf{x}_{T} / \partial \mathbf{q}$, $\operatorname{rank}\left(\mathbf{J}_{x T}\right)=k$ becomes

$$
\dot{\boldsymbol{\sigma}}_{x T}=\mathbf{J}_{x T} \mathbf{M}^{-1}(\boldsymbol{\tau}-\mathbf{b}-\mathbf{g})+\left(\mathbf{G}_{x T} \mathbf{J}_{x T}+\dot{\mathbf{J}}_{x T}\right) \dot{\mathbf{q}}-\dot{\boldsymbol{\xi}}_{x T}^{\mathrm{ref}} .
$$

Since $\operatorname{rank}\left(\mathbf{J}_{x T}\right)=k<n$, the torque can be expressed in the form $\boldsymbol{\tau}=\mathbf{J}_{x T}^{\mathrm{T}} \mathbf{f}_{x T}+\mathbf{N}_{x T}^{\mathbf{T}} \boldsymbol{\tau}_{0}$, where $\mathbf{N}_{x T}^{\mathbf{T}}$ is the projection matrix such that term $\mathbf{N}_{x T}^{\mathrm{T}} \boldsymbol{\tau}_{0}$ does not influence the projection of the system motion into manifold (25). Now, (26) can be expressed as

$$
\begin{aligned}
\dot{\boldsymbol{\sigma}}_{x T}= & \left(\mathbf{J}_{x T} \mathbf{M}^{-1} \mathbf{J}_{x T}^{\mathrm{T}}\right) \mathbf{f}_{x T}+\mathbf{J}_{x T} \mathbf{M}^{-1} \mathbf{N}_{x T}^{\mathrm{T}} \boldsymbol{\tau}_{0} \\
& -\mathbf{J}_{x T} \mathbf{M}^{-1}(\mathbf{b}+\mathbf{g})+\left(\mathbf{G}_{x T} \mathbf{J}_{x T}+\dot{\mathbf{J}}_{x T}\right) \dot{\mathbf{q}}-\dot{\boldsymbol{\xi}}_{x T}^{\mathrm{ref}}
\end{aligned}
$$

By taking $\mathbf{N}_{x T}^{\mathrm{T}}=\left(\mathbf{I}-\mathbf{J}_{x T}^{\mathrm{T}} \tilde{\mathbf{J}}_{x T}^{\mathrm{T}}\right)$ and $\tilde{\mathbf{J}}_{x T}^{\mathrm{T}}=\left(\mathbf{J}_{x T}^{\mathrm{T}} \mathbf{M}^{-1} \mathbf{J}_{x T}^{\mathrm{T}}{ }^{\mathrm{T}}\right)^{-1}$ $\mathbf{J}_{x T}^{\mathrm{T}} \mathbf{M}^{-1}$, the term $\mathbf{J}_{x T} \mathbf{M}^{-1} \mathbf{N}_{x T}^{\mathrm{T}} \boldsymbol{\tau}_{0}=\mathbf{0}$; thus, it does not influence motion (27). The structure of the inverse is the same as the one obtained for constrained system (13). The same structure of inverse is obtained in [17] as a result of the kinetic energy minimization.

The selection of control can follow the same steps as in the case of constrained systems. One can rewrite (27) as

$$
\begin{aligned}
\dot{\boldsymbol{\sigma}}_{x T}= & \mathbf{f}_{x T}^{*}+\mathbf{d}_{x T}, \quad \mathbf{f}_{x T}^{*}=\left(\mathbf{J}_{x T} \mathbf{M}^{-1} \mathbf{J}_{x T}^{\mathrm{T}}\right) \mathbf{f} \\
\mathbf{d}_{x T}= & -\mathbf{J}_{x T} \mathbf{M}^{-1}(\mathbf{b}+\mathbf{g}) \\
& +\left(\mathbf{G}_{x T} \mathbf{J}_{x T}+\dot{\mathbf{J}}_{x T}\right) \dot{\mathbf{q}}-\dot{\boldsymbol{\xi}}_{x T}^{\mathrm{ref}} .
\end{aligned}
$$

By selecting the task control torque in the form

$$
\mathbf{J}_{x T}^{\mathrm{T}} \mathbf{f}_{x T}=-\mathbf{J}_{x T}^{\mathrm{T}}\left(\mathbf{J}_{x T} \mathbf{M}^{-1} \mathbf{J}_{x T}^{\mathrm{T}}\right)^{-1}\left(\hat{\mathbf{d}}_{x T}+\mathbf{D}_{x T} \boldsymbol{\sigma}_{x T}\right)
$$

where $\hat{\mathbf{d}}_{x T}$ is the estimation of disturbance $\mathbf{d}_{x T}$, closed-loop transient is determined as

$$
\begin{aligned}
\dot{\boldsymbol{\sigma}}_{x T}+\mathbf{D}_{x T} \boldsymbol{\sigma}_{x T} & =\ddot{\mathbf{x}}_{T}+\left(\mathbf{G}_{x T}+\mathbf{D}_{x T}\right) \dot{\mathbf{x}}_{T}+\mathbf{G}_{x T} \mathbf{D}_{x T} \mathbf{x}_{T} \\
& =\mathbf{p}_{x T} .
\end{aligned}
$$

The term $\mathbf{p}_{x T}=\mathbf{d}_{x T}-\hat{\mathbf{d}}_{x T}$ is the disturbance estimation error. Transient (30) has the same form as (26) obtained for constrained system; thus, full symmetry between the control of constrained motion and the task control is demonstrated. By inserting $\boldsymbol{\tau}=\mathbf{J}_{x T}^{\mathrm{T}} \mathbf{f}_{x T}+\mathbf{N}_{x T}^{\mathbf{T}} \boldsymbol{\tau}_{0}$ and (30) into (13), the remaining part of the motion of the system can be determined as

$$
\begin{aligned}
\mathbf{M} \ddot{\mathbf{q}}+\mathbf{b}+\mathbf{g}= & \mathbf{N}_{\varphi}^{\mathrm{T}} \boldsymbol{\tau}_{0}+\mathbf{J}_{x T}^{\mathrm{T}}\left(\mathbf{J}_{x T} \mathbf{M}^{-1} \mathbf{J}_{x T}^{\mathrm{T}}\right)^{-1} \\
& \times \mathbf{J}_{x T} \mathbf{M}^{-1}(\mathbf{b}+\mathbf{g})-\mathbf{J}_{x T}^{\mathrm{T}}\left(\mathbf{J}_{x T} \mathbf{M}^{-1} \mathbf{J}_{x T}^{\mathrm{T}}\right)^{-1} \\
& \times\left(\left(\mathbf{G}_{x T} \mathbf{J}_{x T}+\dot{\mathbf{J}}_{x T}\right) \dot{\mathbf{q}}+\mathbf{D}_{x T} \boldsymbol{\sigma}_{x T}\right)
\end{aligned}
$$

\section{Task Control for Constrained Systems}

Let us now discuss the task control problem for system (13) while constraining the constraints. That effectively means controlling system (16) or (24) in such a way that the desired 
task is achieved while constraints $\phi(\mathbf{q})=\mathbf{0} \in \Re^{m}$ with constraint matrix $[\partial \phi(\mathbf{q}) / \partial \mathbf{q}]=\mathbf{J}_{\varphi} \in \Re^{m \times n}$ are maintained. The dimension of the null space is $l=n-m$, and one can define $l=n-m$ independent tasks for the system in such a way that in the overall system, there is no conflict between the task and the constraints. Let the task vector be required to track its smooth reference $\mathbf{x}_{T}^{\text {ref }} \in \Re^{l}$. The velocity vector associated with the task can be expressed as $\dot{\mathbf{x}}_{T}=\left[\partial \mathbf{x}_{T}(\mathbf{q}) / \partial \mathbf{q}\right] \dot{\mathbf{q}}=$ $\mathbf{J}_{x T} \dot{\mathbf{q}}$, where $\mathbf{J}_{x T} \in \Re^{l \times n}$ is assumed to have $\operatorname{rank}\left(\mathbf{J}_{x T}\right)=l$. Let the force vector associated with the task be $\mathbf{f}_{x T} \in \Re^{(n-m)}$, and then, the torque can be expressed as $\boldsymbol{\tau}=\left(\mathbf{J}_{x T} \mathbf{N}_{\phi}\right)^{\mathrm{T}} \mathbf{f}_{x T}+$ $\mathbf{J}_{\varphi}^{\mathrm{T}} \mathbf{f}_{\phi}, \mathbf{N}_{\varphi}^{\mathrm{T}}=\left(\mathbf{I}-\mathbf{J}_{\varphi}^{\mathrm{T}} \tilde{\mathbf{J}}_{\varphi}^{\mathrm{T}}\right)$, and $\tilde{\mathbf{J}}_{\varphi}^{\mathrm{T}}=\left(\mathbf{J}_{\varphi} \mathbf{M}^{-1} \mathbf{J}_{\varphi}^{\mathrm{T}}\right)^{-1} \mathbf{J}_{\varphi} \mathbf{M}^{-1}$. The control input should be found to enforce sliding-mode motion in manifold

$$
S_{x T}=\left\{\mathbf{q}, \dot{\mathbf{q}}: \boldsymbol{\sigma}_{x T}\left(\boldsymbol{\xi}_{T}\left(\mathbf{x}_{T}, \dot{\mathbf{x}}_{T}\right), \boldsymbol{\xi}_{T}^{\mathrm{ref}}\right)=\mathbf{0}\right\}
$$

where $\boldsymbol{\xi}_{T}\left(\mathbf{x}_{T} \dot{\mathbf{x}}_{T}\right), \boldsymbol{\xi}_{T}^{\text {ref }}$ stand for $((n-m) \times 1)$ configuration vector and its reference. This problem is the same as the task control discussed in the previous section. The only change is that now, Jacobian matrix should take into account the constraints, and it can be expressed as $\mathbf{J}_{T}=\mathbf{J}_{x T} \mathbf{N}_{\phi}$. By selecting configuration of the system related to the task as $\boldsymbol{\xi}_{T}=\mathbf{G}_{T} \mathbf{x}_{T}+\dot{\mathbf{x}}_{T}, \mathbf{G}_{T}>0, \boldsymbol{\xi}_{T}^{\mathrm{ref}}=\mathbf{G}_{T} \mathbf{x}_{T}^{\mathrm{ref}}+\dot{\mathbf{x}}_{T}^{\mathrm{ref}}$, and $\operatorname{rank}\left(\mathbf{G}_{T}\right)=l$, the projection of the system with physical constraints (14) in manifold (32) can be expressed in the following form:

$$
\begin{aligned}
\dot{\boldsymbol{\sigma}}_{T}=\mathbf{J}_{T} \mathbf{M}^{-1}(\boldsymbol{\tau}-\mathbf{b}-\mathbf{g} & \left.+\mathbf{J}_{\varphi}^{\mathrm{T}} \boldsymbol{\lambda}\right) \\
& +\left(\mathbf{G}_{T} \mathbf{J}_{T}+\dot{\mathbf{J}}_{T}\right) \dot{\mathbf{q}}-\dot{\boldsymbol{\xi}}_{T}^{\mathrm{ref}} .
\end{aligned}
$$

Since $\operatorname{rank}\left(\mathbf{J}_{T}\right)=l<n$, the torque can be expressed in the form $\boldsymbol{\tau}=\mathbf{N}_{\varphi}^{\mathbf{T}} \mathbf{J}_{x T}^{\mathrm{T}} \mathbf{f}_{x T}+\mathbf{J}_{\varphi}^{\mathrm{T}} \mathbf{f}_{\boldsymbol{\phi}}$, and (33) can be rewritten as

$$
\begin{aligned}
\dot{\boldsymbol{\sigma}}_{T}=\left(\mathbf{J}_{T} \mathbf{M}^{-1} \mathbf{J}_{T}^{\mathrm{T}}\right) \mathbf{f}_{x T}+\mathbf{J}_{T} \mathbf{M}^{-1} \mathbf{J}_{\varphi}^{\mathrm{T}}\left(\mathbf{f}_{\varphi}+\boldsymbol{\lambda}\right) \\
\quad-\mathbf{J}_{T} \mathbf{M}^{-1}(\mathbf{b}+\mathbf{g})+\left(\mathbf{G}_{T} \mathbf{J}_{T}+\dot{\mathbf{J}}_{T}\right) \dot{\mathbf{q}}-\dot{\boldsymbol{\xi}}_{T}^{\mathrm{ref}} .
\end{aligned}
$$

The term $\quad \mathbf{J}_{T} \mathbf{M}^{-1} \mathbf{J}_{\varphi}^{\mathrm{T}}\left(\mathbf{f}_{\varphi}+\boldsymbol{\lambda}\right)=\mathbf{J}_{x T} \mathbf{N}_{\varphi} \mathbf{M}^{-1} \mathbf{J}_{\varphi}^{\mathrm{T}}\left(\mathbf{f}_{\varphi}+\right.$ $\boldsymbol{\lambda})=\mathbf{0}$; thus, (34) does not depend on the constraint force and can be expressed in the following form:

$$
\begin{aligned}
& \dot{\boldsymbol{\sigma}}_{T}=\mathbf{f}_{T}^{*}+\mathbf{d}_{T}, \quad \mathbf{f}_{T}^{*}=\left(\mathbf{J}_{T} \mathbf{M}^{-1} \mathbf{J}_{T}^{\mathrm{T}}\right) \\
& \mathbf{d}_{T}=-\mathbf{J}_{T} \mathbf{M}^{-1}(\mathbf{b}+\mathbf{g})+\left(\mathbf{G}_{T} \mathbf{J}_{T}+\dot{\mathbf{J}}_{T}\right) \dot{\mathbf{q}}-\dot{\boldsymbol{\xi}}_{T}^{\mathrm{ref}} .
\end{aligned}
$$

This structure has the same form as (28); thus, the control issues can be solved in the same way. By selecting the task control torque as

$$
\boldsymbol{\tau}_{T}=\mathbf{J}_{T}^{\mathrm{T}}\left(\mathbf{J}_{T} \mathbf{M}^{-1} \mathbf{J}_{T}^{\mathrm{T}}\right)^{-1}\left(\hat{\mathbf{d}}_{T}+\mathbf{D}_{T} \boldsymbol{\sigma}_{T}\right)
$$

where $\hat{\mathbf{d}}_{T}$ is the estimation of disturbance $\mathbf{d}_{T}$, and the task closed-loop transient is determined as

$$
\begin{aligned}
\dot{\boldsymbol{\sigma}}_{T}+\mathbf{D}_{T} \boldsymbol{\sigma}_{T} & =\ddot{\mathbf{x}}_{T}+\left(\mathbf{G}_{T}+\mathbf{D}_{T}\right) \dot{\mathbf{x}}_{T}+\mathbf{G}_{T} \mathbf{D}_{T} \mathbf{x}_{T} \\
& =\mathbf{d}_{T}-\hat{\mathbf{d}}_{T}
\end{aligned}
$$

the constrained motion is the same as in (22)

$$
\begin{aligned}
\dot{\boldsymbol{\sigma}}_{\varphi}+\mathbf{D}_{\varphi} \boldsymbol{\sigma}_{\varphi} & =\ddot{\boldsymbol{\phi}}+\left(\mathbf{G}_{\varphi}+\mathbf{D}_{\varphi}\right) \dot{\boldsymbol{\phi}}+\mathbf{G}_{\varphi} \mathbf{D}_{\varphi} \boldsymbol{\phi} \\
& =\mathbf{p}_{\varphi}=\mathbf{d}_{\varphi}-\hat{\mathbf{d}}_{\varphi} .
\end{aligned}
$$

Similarly, control can be obtained for system (13) with virtual constraints.

Motion (37) with $\boldsymbol{\sigma}_{T} \in \Re^{n-m=l}$ is describing $(n-m)$ dynamic system, and (38) with $\boldsymbol{\sigma}_{\varphi} \in \Re^{m}$ is describing an $m$ dimensional dynamics; thus, the overall motion is on the order of $(n-m)+m=n$. Both (37) and (38) do not depend on the parameters of the system and are defined by the design parameters. The control input that enforces the constraint and the task has the same structure, and the control error depends on the equivalent control or disturbance estimation error. Equations (37) and (38) are similar to those that can be obtained under disturbance compensation and PD controller [18], [19].

Presented results show that control of multibody mechanical systems can be designed in the sliding mode framework by partitioning the system into blocks of joints associated with certain function of the system. The partition may depend on the evolution of system in time. The full analysis of the possibilities is out of the framework of this paper.

\section{E. Example of Bilateral Systems Control}

Behavior of an ideal bilateral system requires the tracking of the master position by the slave and the forces on master and slave side to be equal but with opposite signs [20], [21]. Assume single DOF systems $S_{i}: m_{i} \ddot{x}_{i}+n_{i}\left(x_{i}, \dot{x}_{i}\right)=f_{i}-g_{\text {iext }}$, $i=m, s$, playing role of the master (index $m$ ) and the slave (index $s$ ). The interaction force with environment on the slave side is $g_{\text {iext }}$. Let $\alpha$ be the position scaling coefficient and $\beta$ be the force scaling coefficient. The force sensed by human operator is $F_{m}=Z_{m} x_{m}=C_{m} x_{m}+Q_{m} \dot{x}_{m}$, where $Z_{m}=$ $C_{m}+Q_{m} s$ stands for the impedance of a human operator. The coefficients $Q_{m}$ and $C_{m}$ can be selected so that impedance perceived by the human operator is shaped in order to give a feeling of a virtual tool in operator's hand. Let $\boldsymbol{\Phi}_{\mathrm{B}}^{\mathrm{T}}=\left[\begin{array}{ll}\zeta_{x} & \zeta_{F}\end{array}\right]$ be the task vector with $\zeta_{x}$ standing for position tracking error and $\zeta_{F}$ standing for force tracking error. The bilateral system operational conditions can be met if the sliding-mode motion is enforced on manifold

$$
S_{\mathrm{B}}=\left\{\left(x_{m}, \mathbf{x}_{s}\right): \boldsymbol{\xi}_{\mathrm{B}}\left(\boldsymbol{\Phi}_{\mathrm{B}}\right)-\boldsymbol{\xi}_{\mathrm{B}}^{\mathrm{ref}}(t)=\boldsymbol{\sigma}_{\mathrm{B}}=0\right\} .
$$

The components of the function vector can be defined as $\zeta_{x}=x_{m}-\alpha x_{s}$ and $\zeta_{F}=Z_{m} x_{m}+\beta g_{s e x t}$, and the corresponding sliding mode manifolds can be defined in the following way:

$$
\begin{gathered}
S_{x}=\left\{\left(x_{m}, x_{s}\right): Q_{x} \dot{\xi}_{x}+C_{x} \xi_{x}=\sigma_{x}=0\right\} \\
S_{F}=\left\{\left(x_{m}, x_{s}\right): Z_{m} x_{m}+\beta g_{\text {sext }}\right. \\
=C_{m} \varepsilon_{F}+Q_{m} \dot{\varepsilon}_{F} \\
-\left(C_{m} x_{s}+Q_{m} \dot{x}_{s}-\beta g_{\text {sext }}\right) \\
\left.=\sigma_{F}=0\right\}
\end{gathered}
$$




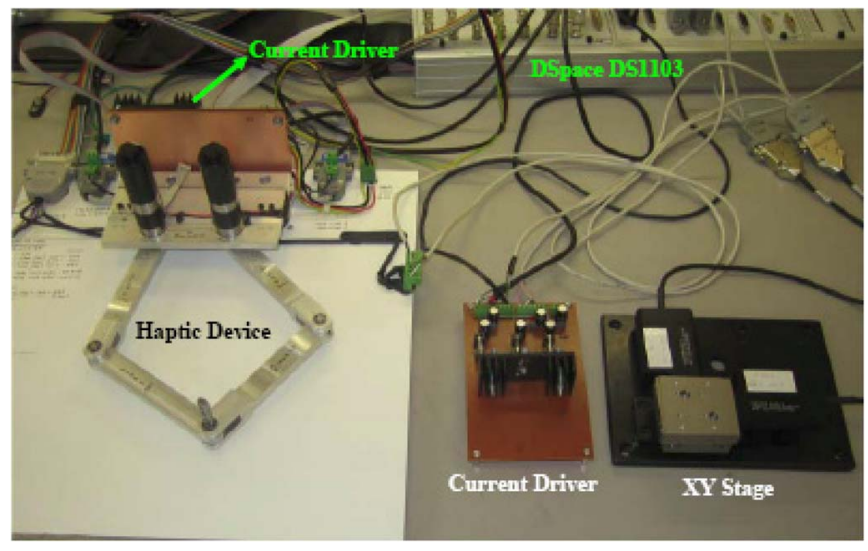

Fig. 2. Experimental system for bilateral control. Master side is on the left, and slave side is on the right part of the image.

$\varepsilon_{F}=x_{m}+x_{s}$ stands for the control error. The projection of the system motion in sliding mode manifolds $S_{x}$ and $S_{F}$ can be described by

$$
\begin{aligned}
\dot{\sigma}_{x}= & f_{x}-d_{x} \\
\dot{\sigma}_{F}= & f_{F}-d_{F} \\
f_{x}= & Q_{x}\left(f_{m} / m_{m}-\alpha f_{s} / m_{s}\right) \\
f_{F}= & Q_{m}\left(f_{m} / m_{m}-\beta f_{s} / m_{s}\right) \\
d_{x}= & \left(n_{m} / m_{m}-\alpha\left(n_{s}+g_{\text {sext }}\right) / m_{s}\right)+C_{x} \dot{\varepsilon}_{x} \\
d_{F}= & \left(n_{m} / m_{m}+\left(n_{s}+g_{\text {sext }}\right) / m_{s}\right) \\
& +\left(\left(Q_{m} \ddot{x}_{s}+C_{m} \dot{x}_{s}\right)-\dot{g}_{\text {sext }}\right) .
\end{aligned}
$$

The formulation of the control problem in (41) is the same as the one described in the previous section-for the task control in constrained system, and thus, the selection of control inputs $f_{x}$ and $f_{F}$ follows the same procedure. Mapping back to $f_{m}$ and $f_{s}$ can be found in (41).

For verification, experimental system consists of the $x-y$ parallel manipulator with Faulhaber 2642012 CR series motors as a master side device and the Cartesian linear microstage with PI M-232.17 actuators in $x$ - and $y$-directions as a slave device. The actuators have a gear ratio of 1:1000, and the position is measured by encoders with 512 ppr. The dSPACE 1103 real-time controller with a $100-\mu$ s measurement sampling rate and a 1-ms control output sampling rate is used. This allows averaging of the measured output. Structure of the overall system is shown in Fig. 2. The position is scaled by factor $x_{m} / x_{s}=(20 / 3)$. The force is not scaled. The forces on the slave system are decoupled (due to the kinematical structure). On the master side, the relation between forces $F_{x}, F_{y}$ and torques is nonlinear.

The controllers on the master side (force control) and on the slave side (position tracking and force limit) are designed, as presented in Section III. The sliding manifolds are selected as $\left(Q_{m} \dot{\varepsilon}_{F}+C_{m} \varepsilon_{F}\right)-\left(\left(Q_{m} \dot{x}_{s}+C_{m} x_{s}\right)-\beta F_{s}\right)=$ $\sigma_{F}, \varepsilon_{F}=x_{m}+x_{s}$, for force control and as $Q_{x} \dot{\varepsilon}_{x}+C_{x} \varepsilon_{x}=$ $\sigma_{x}, \varepsilon_{x}=x_{m}-\alpha x_{s}$, for position tracking. Parameters of the controller are $Q_{m}=25, C_{m}=5000, \alpha=(20 / 3)$, and $\beta=1$. The $x-y$ motion of the master and slave systems is shown in

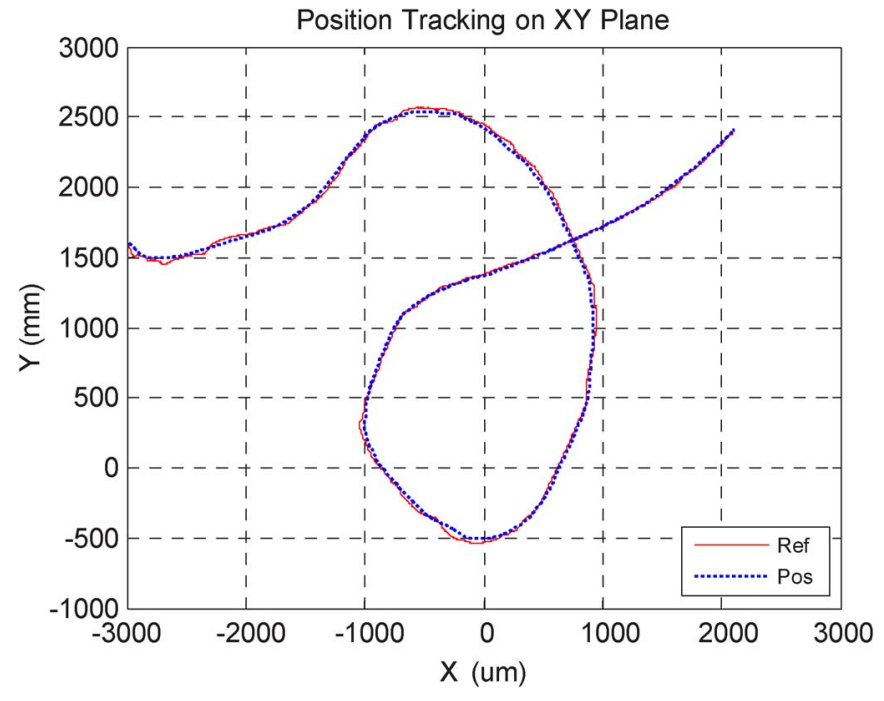

Fig. 3. Position tracking in the master and slave systems in $x-y$ plane.

Fig. 3. The real slave position and the scaled master position are depicted in order to be able to compare them more clearly.

\section{CONCLUSION}

In this paper, application of the SMC in the control of multibody mechanical systems is discussed. The approach leads to a unified formulation of the control of constrained systems and the task control. It has been shown that the same approach can be used in controlling systems in interaction, establishing desired functional relation between systems, and allowing the application of the same framework to bilateral and "function control" systems. This allows one to use the same structure of the controller for all tasks. The structure of the controller is selected to fulfill Lyapunov stability criteria and enforce the sliding mode motion on the sliding mode manifold. The realization of the SMC in the discrete-time framework is discussed, and it is shown that under the proposed control, the motion of the system remains within the boundary layer on the order of $o\left(T^{2}\right)$. Experiments on high-precision PZT-based system with a nonlinear gain and on the bilateral control system are shown.

\section{REFERENCES}

[1] S. Arimoto and P. T. A. Nguyen, "Principle of superposition for realizing dexterous pinching motions of a pair of robot fingers with soft-tips," IEICE Trans. Fundam. Electron. Commun. Comput. Sci., vol. E84-A, no. 1, pp. 39-47, 2001.

[2] D. Lee and P. Y. Li, "Passive bilateral feedforward control of linear dynamically similar teleoperated manipulators," IEEE Trans. Robot. Autom., vol. 19, no. 3, pp. 443-456, Jun. 2003.

[3] T. Tsuji, K. Ohnishi, and A. S̆abanović, "A controller design method based on functionality," IEEE Trans. Ind. Electron., vol. 54, no. 6, pp. 33353343, Dec. 2007.

[4] A. Šabanović and M. Elitas, "SMC based bilateral control," in Proc. Int. Symp. Ind. Electron., Vigo, Spain, Jun. 3-7, 2007, pp. 2144-2149.

[5] D. G. Gersem, "Kinaesthetic feedback and enhanced sensitivity in robotic endoscopic telesurgery," Ph.D. dissertation, Katholieke Universiteit, Leuven, Belgium, Feb. 2005.

[6] G. J. Raju, "Operator adjustable impedance in bilateral remote manipulation," Ph.D. dissertation, Massachusetts Inst. Technol., Cambridge, MA, 1989. 
[7] Y. Strassberg, A. A. Goldenberg, and J. K. Mills, "A new control scheme for bilateral teleoperating systems: Performance evaluation and comparison," in Proc. IEEE/RSJ Int. Conf. Intell. Robots Syst., Jul. 7-10, 1992, vol. 2, pp. 865-872.

[8] S. Katsura and K. Ohishi, "Modal system design of multirobot systems by interaction mode control," IEEE Trans. Ind. Electron., vol. 54, no. 3, pp. 1537-1546, Jun. 2007.

[9] V. I. Utkin, J. Guldner, and J. Shi, Sliding Modes in Electromechanical Systems. New York: Taylor \& Francis, 1999.

[10] S. V. Drakunov and V. I. Utkin, "On discrete-time sliding modes," in Proc. Nonlinear Control Syst. Des. Conf., Capri, Italy, Mar. 1989, pp. 273-278.

[11] K. D. Young, V. I. Utkin, and U. Ozguner, "A control engineer's guide to sliding mode control," IEEE Trans. Control Syst. Technol., vol. 7, no. 3, pp. 328-342, May 1999.

[12] B. Drazenovic, "The invariance conditions in variable structure systems," Automatica, vol. 5, no. 3, pp. 287-295, May 1969.

[13] K. Ohnishi, M. Shibata, and T. Murakami, "Motion control for advanced mechatronics," IEEE/ASME Trans. Mechatronics, vol. 1, no. 1, pp. 56-67, Mar. 1996.

[14] R. De Carlo and S. Drakunov, "Sliding mode control design via Lyapunov approach," in Proc. 33rd Conf. Decision Control, Lake Buena Vista, FL, Dec. 1994, pp. 1925-1930.

[15] V. I. Utkin and H. Lee, "The Chattering Analysis," in Proc. 12th EPEPEMC, Portoroz, Slovenia, Aug. 31-Dec. 1, 2006, pp. 2014-2019.

[16] A. Šabanović, N. Šabanović, and K. Jezernik, "Sliding modes in sampleddata systems," Automatika, vol. 44, pp. 3-4, 2003. Zagreb.

[17] V. De Sapiro and O. Khatib, "Operational space control of multibody systems with explicit holonomic constraints," in Proc. IEEE IROS Conf., Barcelona, Spain, Apr. 2005, pp. 2950-2956.

[18] S. Katsura and K. Ohnishi, "A realization of haptic training system by multilateral control," IEEE Trans. Ind. Electron., vol. 53, no. 6, pp. 19351942, Dec. 2006.

[19] S. Katsura and K. Ohishi, "Modal system design of multirobot systems by interaction mode control," IEEE Trans. Ind. Electron., vol. 54, no. 3, pp. 1537-1546, Jun. 2007.

[20] T. Tsuji, K. Natori, and K. Ohnishi, "A controller design method of bilateral control system," in Proc. EPE-PEMC, 2004, vol. 4, pp. 123-128.

[21] T. Tsuji, "Motion control for adaptation to human environment," Ph.D. dissertation, Keio Univ., Tokyo, Japan, 2005.

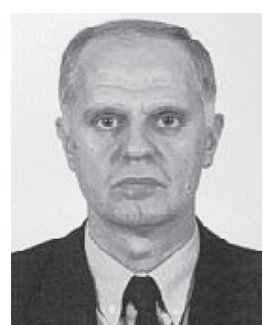

Asif S̆abanović (M'85-SM'03) received the B.S., M.S., and Dr.Sci. degrees in electrical engineering from the University of Sarajevo, Bosnia and Herzegovina, in 1970, 1975, and 1979, respectively.

Previously, he had been with the University of Sarajevo. He was a Visiting Professor with Caltech, Pasadena, CA; Keio University, Yokohama, Japan; and Yamaguchi University, Yamaguchi, Japan. He was the Head of the CAD/CAM and Robotics Department, Tubitak-MAM, Turkey. He is currently with Sabanci University, Istanbul, Turkey. His fields of research interest include power electronics, sliding-mode control, motion control, and mechatronics.

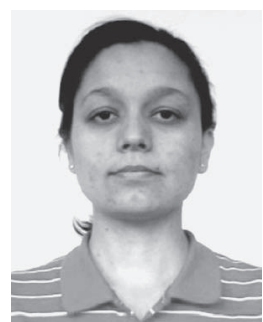

Meltem Elitas received the B.S. degree in electrical engineering from Yildiz Technical University, Istanbul, Turkey, in 2005, and the M.S. degree in mechatronics from Sabanci University, Istanbul, in 2007. She is currently working toward the Ph.D. degree in the School of Life Sciences, École Polytechnique Fédérale de Lausanne, Lausanne, Switzerland.

Her research interests include motion control systems, microfluidics, microfabrication, and mechanistic basis of bacterial persistence.

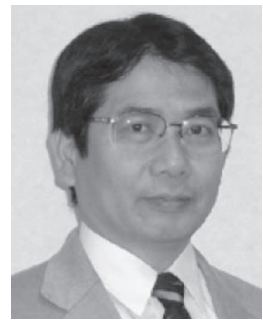

Kouhei Ohnishi (S'78-M'80-SM'00-F'01) received the B.E., M.E., and Ph.D. degrees in electrical engineering from the University of Tokyo, Tokyo, Japan, in 1975, 1977, and 1980, respectively.

Since 1980, he has been with Keio University, Yokohama, Japan. His research interests include power electronics, mechatronics, motion control, and haptics.

Prof. Ohnishi was the recipient of Best Paper Awards from the Institute of Electrical Engineers of Japan and the Japan Society for Precision Engineering. He was also the recipient of the Dr.-Ing. Eugene Mittelmann Achievement Award from the IEEE Industrial Electronics Society in 2004. 